\title{
Optimization of fuzzy inventory model for EOQ using Lagrangian method
}

\author{
K. Kalaiarasi ${ }^{1}$, M. Sumathi ${ }^{2}$ and H. Mary Henrietta ${ }^{3^{*}}$
}

\begin{abstract}
This paper discusses the existence of defective items in a manufacturing process. A rework strategy is implemented to rectify the defective items. A rework cost function under fuzzy environment which includes both synchronous and asynchronous items. The paper develops fuzzy optimal total cost function and fuzzy production quantity using trapezoidal numbers and applying Lagrangian method. A numerical example follows to justify the solution procedure.

Keywords

Economic order quantity (EOQ), Fuzzy inventory, Graded mean integration and Lagrangian method.

AMS Subject Classification

$65 \mathrm{~K} 10$.

${ }^{1}$ Department of Mathematics, Cauvery College for Women, Trichy-620018, Tamil Nadu, India.

${ }^{2}$ PG and Research Department of Mathematics, Khadir Mohideen College (Affiliated to Bharathidasan University), Adhirampattinam-614701, Tamil Nadu, India.

${ }^{3}$ Research Scholar (Part-Time), PG and Research Department of Mathematics, Khadir Mohideen College (Affiliated to Bharathidasan University), Adhirampattinam-614701, Tamil Nadu, India; Department of Mathematics, Saveetha Engineering College, Chennai-602105, Tamil Nadu, India.

*Corresponding author: ${ }^{3}$ mary.henriet123@gmail.com

Article History: Received 24 March 2019; Accepted 17 July 2019

(C)2019 MJM.
\end{abstract}

\section{Contents}

1 Introduction .................................. 497

2 Notations .................................. 497

3 Preliminaries $\ldots \ldots \ldots \ldots \ldots \ldots \ldots \ldots \ldots \ldots \ldots, 498$

3.1 The Fuzzy Arithmetical Operations . . . . . . 498

3.2 Extension of the Lagrangian method . . . . . . 498

3.3 Graded mean integration representation method . 498

4 Fuzzy Integrated Inventory Model for Crisp produc-

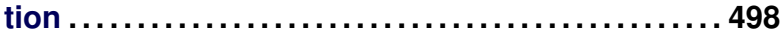

5 Fuzzy integrated inventory model for fuzzy EOQ.499

6 Conclusion .................................... 501

References ............................. 501

\section{Introduction}

In inventory management system, EOQ is the economic order quantity that minimizes the holding costs and ordering costs. Ford W. Harris[4] developed this model but it is R.H. Wilson [13] who had applied it extensively. Zadeh [14] in the year 1965 had introduced the fuzzy set theory that dealt with uncertainty and also his application of fuzzy set theory in the field of production management plays a vital role. Chen et al. [1,2] studied the back-order fuzzy inventory model under function principle and graded mean integration representations of generalized fuzzy number respectively. Presently, Muhammad Al-Salamah [7], analyzed the economic production quantity in an imperfect manufacturing process with synchronous and asynchronous flexible rework rates. For more details on this theory and on its applications, we suggest the reader to refer $[3,5,6,8-12,15,16]$.

Inspired by the above mentioned works, in this paper, we have considered trapezoidal fuzzy numbers and optimization method is carried out by Lagrangian method. For defuzzifying the total cost graded mean integration method has been applied. Finally, numerical examples highlight the contrast between the crisp and fuzzy scenarios.

\section{Notations}

$\kappa:$ Production set up cost

$P$ : Production rate (items per time, $P>D$ )

$T$ : Cycle length 
$B$ : Backorder time

$Q:$ lot size (items)

$A T C(Q, B ; P R)$ : average total cost per unit of time

\section{Preliminaries}

Definition 3.1. A fuzzy set $\tilde{A}$ defined on $R[\infty,-\infty]$, if the membership function of $\tilde{A}$ is defined by

$$
\mu_{\tilde{A}}(x)= \begin{cases}1, & x \in A \\ 0, & x \notin A\end{cases}
$$

\subsection{The Fuzzy Arithmetical Operations}

Function principle is proposed to be as the fuzzy arithmetical operations by trapezoidal fuzzy numbers. Suppose $\tilde{A}=\left(x_{1}, x_{2}, x_{3}, x_{4}\right)$ and $\tilde{B}=\left(y_{1}, y_{2}, y_{3}, y_{4}\right)$ be two trapezoidal fuzzy numbers. Then

(1)The addition of $\tilde{A}$ and $\tilde{B}$ is

$$
\tilde{A} \oplus \tilde{B}=\left(x_{1}+y_{1}, x_{2}+y_{2}, x_{3}+y_{3}, x_{4}+y_{4}\right),
$$

where $x_{1}, x_{2}, x_{3}, x_{4}, y_{1}, y_{2}, y_{3}$, and $y_{4}$ are any real numbers.

(2) The multiplication of $\tilde{A}$ and $\tilde{B}$ is

$$
\tilde{A} \otimes \tilde{B}=\left(C_{1}, C_{2}, C_{3}, C_{4}\right),
$$

where $Z_{1}=\left\{x_{1} y_{1}, x_{1} y_{4}, x_{4} y_{1}, x_{4} y_{4}\right\}, Z_{2}=\left\{x_{2} y_{2}, x_{2} y_{3}, x_{3} y_{2}, x_{3} y_{3}\right\}$, $C_{1}=\min Z_{1}, C_{2}=\min Z_{2}, C_{3}=\max Z_{1}, C_{4}=\max Z_{2}$.

If $x_{1}, x_{2}, x_{3}, x_{4}, y_{1}, y_{2}, y_{3}$, and $y_{4}$ are all zero positive real numbers then

$$
\tilde{A} \otimes \tilde{B}=\left(x_{1} y_{1}, x_{2} y_{2}, x_{3} y_{3}, x_{4} y_{4}\right) \text {. }
$$

(3) The subtraction of $\tilde{A}$ and $\tilde{B}$ is

$$
\tilde{A} \ominus \tilde{A}=\left(x_{1}-y_{4}, x_{2}-y_{3}, x_{3}-y_{2}, x_{4}-y_{1}\right),
$$

where $-\tilde{B}=\left(-y_{4},-y_{3},-y_{2},-y_{1}\right)$, also $x_{1}, x_{2}, x_{3}, x_{4}, y_{1}, y_{2}, y_{3}$ and $y_{4}$ are any real numbers.

(4) The division of $\tilde{A}$ and $\tilde{B}$ is

$$
\tilde{A} \oslash \tilde{B}=\left(\frac{x_{1}}{y_{4}}, \frac{x_{2}}{y_{3}}, \frac{x_{3}}{y_{2}}, \frac{x_{4}}{y_{1}}\right),
$$

where $\frac{1}{\tilde{B}}=\tilde{B}^{-1}=\left(\frac{1}{y_{4}}, \frac{1}{y_{3}}, \frac{1}{y_{2}}, \frac{1}{y_{1}}\right), y_{1}, y_{2}, y_{3}$ and $y_{4}$ are positive real numbers. Also $x_{1}, x_{2}, x_{3}, x_{4}, y_{1}, y_{2}, y_{3}$ and $y_{4}$ are nonzero positive numbers.

(5) For any $\alpha \in R$,

(a) If $\alpha \geq 0$, then $\alpha \otimes \tilde{A}=\left(\alpha a_{1}, \alpha a_{2}, \alpha a_{3}, \alpha a_{4}\right)$.

(b) If $\alpha<0$, then $\alpha \otimes \tilde{A}=\left(\alpha a_{4}, \alpha a_{3}, \alpha a_{2}, \alpha a_{1}\right)$.

\subsection{Extension of the Lagrangian method}

Solving a nonlinear programming problem by obtaining the optimum solution was discussed by Taha with equality constraints, also by solving those inequality constraints using Lagrangian method.

Suppose if the problem is given as $\min y=f(x)$

Subject to $g_{i}(x) \geq 0, i=1,2, \ldots, m$.

The constraints are non-negative say $x \geq 0$ if included in the $m$ constraints. Then the procedure of the extension of the Lagrangian method will involve the following steps.

Step 1: Solve the unconstrained problem $\min y=f(x)$

If the resulting optimum satisfies all the constraints, then stop since all the constraints are inessential or else set $K=1$ and move to step 2 .

Step 2: Activate any $K$ constraints (i.e., convert them into equalities) and optimize $f(x)$ subject to the $K$ active constraints by the Lagrangian method. If the resulting solution is feasible with respect to the remaining constraints, we shall repeat the steps. If all sets of active constraints taken $K$ at a time are considered without confront a feasible solution, go to step 3 .

Step 3: If $K=m$, stop; there's no feasible solution, otherwise set $K=K+1$ and go to step 2 .

\subsection{Graded mean integration representation method}

Graded mean integration representation method was introduced by Chen and Hsieh [2] based on the integral value of graded mean $h$-level of generalized fuzzy number for defuzzifying generalized fuzzy number. First we define a generalized fuzzy number as follows:

$\tilde{A}=\left(a_{1}, a_{2}, a_{3}, a_{4},\right)_{L R}$.

By graded mean integration are the inverses of $L$ and $R$ are $L^{-1}$ and $R^{-1}$ respectively. The graded mean $h$-level value of the generalized fuzzy number $\tilde{A}=\left(a_{1}, a_{2}, a_{3}, a_{4}\right)_{L R}$ is given by $\frac{h}{2}\left[L^{-1}(h)+R^{-1}(h)\right]$. Then the graded mean integration representation of $P(\tilde{A})$ with grade then

$$
P(\tilde{A})=\frac{\int_{0}^{\omega_{A}} \frac{h}{2}\left[L^{-1}(h)+R^{-1}(h)\right] d h}{\int_{0}^{\omega_{A}} h d h},
$$

where $0<h \leq \omega_{A}$ and $0<\omega_{A} \leq 1$.

In this paper, we have used trapezoidal fuzzy numbers as fuzzy parameters for the production inventory model. Let $\tilde{B}=\left(b_{1}, b_{2}, b_{3}, b_{4}\right)$. Then the graded mean integration representation is given by the formula as

$$
\begin{aligned}
P(\tilde{B}) & =\frac{\int_{0}^{1} \frac{h}{2}\left[\left(b_{1}+b_{4}\right)+h\left(b_{2}-b_{1}-b_{4}+b_{3}\right)\right] d h}{\int_{0}^{1} h d h} \\
& =\frac{b_{1}+2 b_{2}+2 b_{3}+b_{4}}{6} .
\end{aligned}
$$

\section{Fuzzy Integrated Inventory Model for Crisp production}

$$
A T C(Q ; B ; P)=\beta-\kappa B+\frac{\nu B^{2}}{Q}+\eta \frac{1}{Q}+\frac{\delta B}{Q}+\chi Q
$$


where $v>0, \delta>0$ and $\eta>0$.

Differentiating partially with respect to $Q$ and equating it to zero we arrive as follows

$$
\begin{array}{r}
\frac{\partial A T C(Q, B ; P)}{\partial Q}=\frac{-v B^{2}}{Q^{2}}-\eta \frac{1}{Q^{2}}-\frac{\delta B}{Q^{2}}+\chi \\
-\frac{v B^{2}}{Q^{2}}-\eta \frac{1}{Q^{2}}-\frac{\delta B}{Q^{2}}+\chi=0
\end{array}
$$

implies the EOQ is

$$
Q=\sqrt{\frac{v B^{2}+\eta+\delta B}{\chi}} .
$$

\section{Fuzzy integrated inventory model for fuzzy EOQ}

The annual integrated total inventory cost is

$$
\operatorname{ATC}(Q, B ; P)=\beta-\kappa B+\frac{v B^{2}}{Q}+\eta \frac{1}{Q}+\frac{\delta B}{Q}+\chi Q .
$$

Suppose

$$
\begin{aligned}
\tilde{Q} & =\left(Q_{1}, Q_{2}, Q_{3}, Q_{4}\right) \\
\tilde{v} & =\left(v_{1}, v_{2}, v_{3}, v_{4}\right) \\
\tilde{\kappa} & =\left(\kappa_{1}, \kappa_{2}, \kappa_{3}, \kappa_{4}\right) \\
\tilde{\beta} & =\left(\beta_{1}, \beta_{2}, \beta_{3}, \beta_{4}\right) \\
\tilde{B} & =\left(B_{1}, B_{2}, B_{3}, B_{4}\right) \\
\tilde{\eta} & =\left(\eta_{1}, \eta_{2}, \eta_{3}, \eta_{4}\right) \\
\tilde{\delta} & =\left(\delta_{1}, \delta_{2}, \delta_{3}, \delta_{4}\right)
\end{aligned}
$$

and

$$
\begin{aligned}
\tilde{\chi}= & \left(\chi_{1}, \chi_{2}, \chi_{3}, \chi_{4}\right) \\
= & \frac{1}{6}\left\{\left[\beta_{1}-\kappa_{4} B_{4}+\frac{v_{1} B_{1}^{2}}{Q}+\frac{\eta_{1}}{Q}+\frac{\delta_{1} B_{1}}{Q}+\chi_{1} Q\right]\right. \\
& +2\left[\beta_{2}-\kappa_{3} B_{3}+\frac{v_{2} B_{2}^{2}}{Q}+\frac{\eta_{2}}{Q}+\frac{\delta_{2} B_{2}}{Q}+\chi_{2} Q\right] \\
& +2\left[\beta_{3}-\kappa_{2} B_{2}+\frac{v_{3} B_{3}^{2}}{Q}+\frac{\eta_{3}}{Q}+\frac{\delta_{3} B_{3}}{Q}+\chi_{3} Q\right] \\
& \left.+\left[\beta_{4}-\kappa_{1} B_{1}+\frac{v_{4} B_{4}^{2}}{Q}+\frac{\eta_{4}}{Q}+\frac{\delta_{4} B_{4}}{Q}+\chi_{4} Q\right]\right\} .
\end{aligned}
$$

Partially differentiating w.r.t $Q$ and equating to zero,

$$
\begin{aligned}
= & \frac{1}{6}\left\{\left[-\frac{\nu_{1} B_{1}^{2}}{Q^{2}}-\frac{\eta_{1}}{Q^{2}}-\frac{\delta_{1} B_{1}}{Q^{2}}+\chi_{1}\right]\right. \\
& +2\left[-\frac{\nu_{2} B_{2}^{2}}{Q^{2}}-\frac{\eta_{2}}{Q^{2}}-\frac{\delta_{2} B_{2}}{Q^{2}}+\chi_{2}\right] \\
& +2\left[-\frac{\nu_{3} B_{3}^{2}}{Q^{2}}-\frac{\eta_{3}}{Q^{2}}-\frac{\delta_{3} B_{3}}{Q^{2}}+\chi_{3}\right] \\
& \left.\left.+\left[-\frac{\nu_{4} B_{4}^{2}}{Q^{2}}-\frac{\eta_{4}}{Q^{2}}-\frac{\delta_{4} B_{4}}{Q^{2}}+\chi_{4}\right]\right]\right\}=0 .
\end{aligned}
$$

Therefore,

$$
\begin{aligned}
Q= & \sqrt{\frac{\left(v_{1}^{2} B_{1}^{2}+2 v_{2} B_{2}^{2}+2 v_{3} B_{3}^{2}+v_{4} B_{4}^{2}\right)+\left(\eta_{1}+2 \eta_{2}+2 \eta_{3}+\eta_{4}\right)+\left(\delta_{1} B_{1}+2 \delta_{2} B_{2}+2 \delta_{3} B_{3}+\delta_{4} B_{4}\right)}{\left(\chi_{1}+2 \chi_{2}+2 \chi_{3}+\chi_{4}\right)}} \\
A T C(Q, B ; P)= & \frac{1}{6}\left\{\left[\beta_{1}-\kappa_{4} B_{4}+\frac{v_{1} B_{1}^{2}}{Q_{4}}+\frac{\eta_{1}}{Q_{4}}+\frac{\delta_{1} B_{1}}{Q_{4}}+\chi_{1} Q_{1}\right]+2\left[\beta_{2}-\kappa_{3} B_{3}+\frac{\nu_{2} B_{2}^{2}}{Q_{3}}+\frac{\eta_{2}}{Q_{4}}+\frac{\delta_{2} B_{2}}{Q_{4}}+\chi_{2} Q_{2}\right]\right. \\
& \left.+2\left[\beta_{3}-\kappa_{2} B_{2}+\frac{v_{3} B_{3}^{2}}{Q_{2}}+\frac{\eta_{3}}{Q_{2}}+\frac{\delta_{3} B_{3}}{Q_{2}}+\chi_{3} Q_{3}\right]+\left[\beta_{4}-\kappa_{1} B_{1}+\frac{v_{4} B_{4}^{2}}{Q_{1}}+\frac{\eta_{4}}{Q_{1}}+\frac{\delta_{4} B_{4}}{Q_{1}}+\chi_{4} Q_{4}\right]\right\} .
\end{aligned}
$$

Solving the non-constraint problem, now we partially differ- $\quad$ Letting $\frac{\partial P}{\partial Q_{2}}=0$.

entiating w.r.t $Q_{1}, Q_{2}, Q_{3}, Q_{4}$ respectively,

$$
\frac{\partial P}{\partial Q_{1}}=\frac{1}{6}\left[\chi_{1}-\frac{v_{4} B_{4}^{2}}{Q_{1}^{2}}-\frac{\eta_{4}}{Q_{1}^{2}}-\frac{\delta_{4} B_{4}}{Q_{1}^{2}}\right]
$$

Letting $\frac{\partial P}{\partial Q_{1}}=0$.

$$
\begin{aligned}
Q_{1} & =\sqrt{\frac{v_{4} B_{4}^{2}+\eta_{4}+\delta_{4} B_{4}}{\chi_{1}}} \\
\frac{\partial P}{\partial Q_{2}} & =\frac{1}{6}\left[2 \chi_{2}-\frac{2 v_{3} B_{3}^{2}}{Q_{2}^{2}}-\frac{2 \eta_{3}}{Q_{2}^{2}}-\frac{2 \delta_{3} B_{3}}{Q_{2}^{2}}\right]
\end{aligned}
$$$$
Q_{2}=\sqrt{\frac{v_{3} B_{3}^{2}+\eta_{3}+\delta_{3} B_{3}}{\chi_{2}}}
$$$$
\frac{\partial P}{\partial Q_{3}}=\frac{1}{6}\left[2 \chi_{3}-\frac{2 v_{2} B_{2}^{2}}{Q_{3}^{2}}-\frac{2 \eta_{2}}{Q_{3}^{2}}-\frac{2 \delta_{2} B_{2}}{Q_{3}^{2}}\right] .
$$ 
Letting $\frac{\partial P}{\partial Q_{3}}=0$.

$$
\begin{aligned}
Q_{3} & =\sqrt{\frac{\nu_{2} B_{2}^{2}+\eta_{2}+\delta_{2} B_{2}}{\chi_{3}}} \\
\frac{\partial P}{\partial Q_{4}} & =\frac{1}{6}\left[\chi_{4}-\frac{\nu_{1} B_{1}^{2}}{Q_{4}^{2}}-\frac{\eta_{1}}{Q_{4}^{2}}-\frac{2 \delta_{1} B_{1}}{Q_{4}^{2}}\right] .
\end{aligned}
$$

Letting $\frac{\partial P}{\partial Q_{4}}=0$.

$$
Q_{4}=\sqrt{\frac{\nu_{1} B_{1}^{2}+\eta_{1}+\delta_{1} B_{1}}{\chi_{4}}}
$$

The above results shows that $Q_{1}>Q_{2}>Q_{3}>Q_{4}$, but contrastingly we have $0<Q_{1} \leq Q_{2} \leq Q_{3} \leq Q_{4}$. Hence $w$ and set $k=1$ and we convert the inequality constraint by optimizing the total cost subject to Lagrangian method subject to $Q_{2}-Q_{1}=0$.

$$
L\left(Q_{1}, Q_{2}, Q_{3}, Q_{4}, \lambda\right)=P(A T C(Q, B, P))-\lambda\left(Q_{2}-Q_{1}\right) .
$$

Now taking the partial derivatives w.r.t $Q_{1}, Q_{2}, Q_{3}, Q_{4}$ and $\lambda$ and the minimize $L\left(Q_{1}, Q_{2}, Q_{3}, Q_{4}, \lambda\right)$, we have

$$
\begin{aligned}
& \frac{\partial L}{\partial Q_{1}}=0 \\
& \frac{1}{6}\left[\chi_{1}-\frac{\nu_{4} B_{4}^{2}}{Q_{1}^{2}}-\frac{\eta_{4}}{Q_{1}^{2}}-\frac{\delta_{4} B_{4}}{Q_{1}^{2}}\right]+\lambda=0 \\
& \frac{\partial L}{\partial Q_{2}}=0 \\
& \frac{1}{6}\left[2 \chi_{2}-\frac{2 v_{3} B_{3}^{2}}{Q_{2}^{2}}-\frac{2 \eta_{3}}{Q_{2}^{2}}-\frac{2 \delta_{3} B_{3}}{Q_{2}^{2}}\right]-\lambda=0 \\
& \frac{\partial L}{\partial Q_{3}}=0 \\
& \frac{1}{6}\left[2 \chi_{3}-\frac{2 v_{2} B_{2}^{2}}{Q_{3}^{2}}-\frac{2 \eta_{2}}{Q_{3}^{2}}-\frac{2 \delta_{2} B_{2}}{Q_{3}^{2}}\right]=0 \\
& \frac{\partial L}{\partial Q_{4}}=0 \\
& \frac{1}{6}\left[\chi_{4}-\frac{v_{1} B_{1}^{2}}{Q_{4}^{2}}-\frac{\eta_{1}}{Q_{4}^{2}}-\frac{\delta_{1} B_{1}}{Q_{4}^{2}}\right]=0 . \\
& \frac{\partial L}{\partial \lambda}=-\left(Q_{2}-Q_{2}\right) \\
& Q_{1}=Q_{2}=\sqrt{\frac{\left(v_{4} B_{4}^{2}+v_{3} B_{3}^{2}\right)+\left(\eta_{4}+\eta_{3}\right)+\left(\delta_{4} B_{4}+\delta_{3} B_{3}\right)}{\chi_{1}+2 \chi_{2}}} \\
& Q_{3}=\sqrt{\frac{v_{2} B_{2}^{2}+\eta_{2}+\delta_{2} B_{2}}{\chi_{3}}} \\
& Q_{4}=\sqrt{\frac{v_{1} B_{1}^{2}+\eta_{1}+\delta_{1} B_{1}}{\chi_{4}}}
\end{aligned}
$$

Now converting the inequality constraints $Q_{2}-Q_{1} \geq 0, Q_{3}-$ $Q_{2} \geq 0$ into equality constraints $Q_{2}-Q_{1}=0$ and $Q_{3}-Q_{2}=0$.
Optimizing $L\left(Q_{1}, Q_{2}, Q_{3}, Q_{4}, \lambda_{1}, \lambda_{2}\right)=P(A T C(Q, B, P))-$ $\lambda_{1}\left(Q_{2}-Q_{1}\right)-\lambda_{2}\left(Q_{3}-Q_{2}\right)$.

$$
\begin{aligned}
\frac{\partial L}{\partial Q_{1}} & =0 \\
\frac{1}{6}\left[\chi_{1}-\frac{v_{4} B_{4}^{2}}{Q_{1}^{2}}-\frac{\eta_{4}}{Q_{1}^{2}}-\frac{\delta_{4} B_{4}}{Q_{1}^{2}}\right]+\lambda_{1} & =0 \\
\frac{\partial L}{\partial Q_{2}} & =0 \\
\frac{1}{6}\left[2 \chi_{2}-\frac{2 v_{3} B_{3}^{2}}{Q_{2}^{2}}-\frac{2 \eta_{3}}{Q_{2}^{2}}-\frac{2 \delta_{3} B_{3}}{Q_{2}^{2}}\right]-\lambda_{1}+\lambda_{2} & =0 \\
\frac{\partial L}{\partial Q_{3}} & =0 \\
\frac{1}{6}\left[2 \chi_{3}-\frac{2 v_{2} B_{2}^{2}}{Q_{3}^{2}}-\frac{2 \eta_{2}}{Q_{3}^{2}}-\frac{2 \delta_{2} B_{2}}{Q_{3}^{2}}\right]-\lambda_{2} & =0 \\
\frac{\partial L}{\partial Q_{4}} & =0 \\
\frac{1}{6}\left[\chi_{4}-\frac{v_{1} B_{1}^{2}}{Q_{4}^{2}}-\frac{\eta_{1}}{Q_{4}^{2}}-\frac{\delta_{1} B_{1}}{Q_{4}^{2}}\right] & =0 \\
\frac{\partial L}{\partial \lambda_{1}=-\left(Q_{2}-Q_{1}\right)} & =0 \\
\frac{\partial L}{\partial \lambda_{2}}=-\left(Q_{3}-Q_{2}\right) & \\
Q_{1}=Q_{2}=Q_{3}=\sqrt{\frac{\left(v_{4} B_{4}^{2}+v_{3} B_{3}^{2}+v_{2} B_{2}^{2}\right)+\left(\eta_{4}+\eta_{3}+\eta_{2}\right)+\left(\delta_{4} B_{4}+\delta_{3} B_{3}+\delta_{2} B_{2}\right)}{\chi_{1}+2 \chi_{2}+2 \chi_{3}}} & \\
Q_{4}=\sqrt{\frac{v_{1} B_{1}^{2}+\eta_{1}+\delta_{1} B_{1}}{\chi_{4}}} &
\end{aligned}
$$

Again converting the inequality constraints $Q_{2}-Q_{1} \geq$ $0, Q_{3}-Q_{2} \geq 0$ and $Q_{4}-Q_{3} \geq 0$ into equality constraints by $Q_{2}-Q_{1}=0, Q_{3}-Q_{2}=0$ and $Q_{4}-Q_{3}=0$ using Lagrangian method. The Lagrangian function is given by

$$
\begin{aligned}
L\left(Q_{1}, Q_{2}, Q_{3}, Q_{4}, \lambda_{1}, \lambda_{2}, \lambda_{3}\right)=P(A T C(Q, B, P)) & \\
-\lambda_{1}\left(Q_{2}-Q_{1}\right)-\lambda_{2}\left(Q_{3}-Q_{2}\right)-\lambda_{3}\left(Q_{4}-Q_{3}\right) & . \\
\frac{\partial L}{\partial Q_{1}} & =0 \\
\frac{1}{6}\left[\chi_{1}-\frac{v_{4} B_{4}^{2}}{Q_{1}^{2}}-\frac{\eta_{4}}{Q_{1}^{2}}-\frac{\delta_{4} B_{4}}{Q_{1}^{2}}\right]+\lambda_{1} & =0 \\
\frac{\partial L}{\partial Q_{2}} & =0 \\
\frac{1}{6}\left[2 \chi_{2}-\frac{2 v_{3} B_{3}^{2}}{Q_{2}^{2}}-\frac{2 \eta_{3}}{Q_{2}^{2}}-\frac{2 \delta_{3} B_{3}}{Q_{2}^{2}}\right]-\lambda_{1}+\lambda_{2} & =0 \\
\frac{\partial L}{\partial Q_{3}} & =0 \\
\frac{1}{6}\left[2 \chi_{3}-\frac{2 v_{2} B_{2}^{2}}{Q_{3}^{2}}-\frac{2 \eta_{2}}{Q_{3}^{2}}-\frac{2 \delta_{2} B_{2}}{Q_{3}^{2}}\right]-\lambda_{2}+\lambda_{3} & =0
\end{aligned}
$$




$$
\begin{aligned}
& \frac{\partial L}{\partial Q_{4}}=0 \\
& \frac{1}{6}\left[\chi_{4}-\frac{v_{1} B_{1}^{2}}{Q_{4}^{2}}-\frac{\eta_{1}}{Q_{4}^{2}}-\frac{\delta_{1} B_{1}}{Q_{4}^{2}}\right]-\lambda_{3}=0 \\
& \frac{\partial L}{\partial \lambda_{1}}=-\left(Q_{2}-Q_{1}\right) \\
& \frac{\partial L}{\partial \lambda_{2}}=-\left(Q_{3}-Q_{2}\right) \\
& \frac{\partial L}{\partial \lambda_{3}}=-\left(Q_{4}-Q_{3}\right) \\
& Q_{1}=Q_{2}=Q_{3}=Q_{4} \\
&=\sqrt{\frac{\left(v_{4} B_{4}^{2}+v_{3} B_{3}^{2}+v_{2} B_{2}^{2}+v_{1} B_{1}^{2}\right)+\left(\eta_{4}+\eta_{3}+\eta_{2}+\eta_{1}\right)+\left(\delta_{4} B_{4}+\delta_{3} B_{3}+\delta_{2} B_{2}+\delta_{1} B_{1}\right)}{\chi_{1}+2 \chi_{2}+2 \chi_{3}+\chi_{4}}}
\end{aligned}
$$

Therefore $\tilde{Q}=\left(Q_{1}, Q_{2}, Q_{3}, Q_{4}\right)$ satisfies all the inequality constraints and we obtain the optimum solution for the problems. Let $Q_{1}=Q_{2}=Q_{3}=Q_{4}=\tilde{Q}^{*}$. Then optimal fuzzy $E O Q$ is given by

$$
\tilde{Q}^{*}=\sqrt{\frac{\left(v_{4} B_{4}^{2}+v_{3} B_{3}^{2}+v_{2} B_{2}^{2}+v_{1} B_{1}^{2}\right)+\left(\eta_{4}+\eta_{3}+\eta_{2}+\eta_{1}\right)+\left(\delta_{4} B_{4}+\delta_{3} B_{3}+\delta_{2} B_{2}+\delta_{1} B_{1}\right)}{\chi_{1}+2 \chi_{2}+2 \chi_{3}+\chi_{4}}} .
$$

\section{Conclusion}

Hereby this paper develops an inventory model with backorders using fuzzy parameters as decision variables. Hence the economic order quantity for the inventory system is derived by minimizing the total cost inventory function. The notations are taken as trapezoidal fuzzy numbers, Lagrangian method has been applied and defuzzification has been done by graded mean representation method. The numerical examples are contingently showcasing the difference between increased crisp cases against the fuzzy model.

\section{References}

[1] S.H. Chen, C.C. Wang and Arthur Ramer, Back-order fuzzy inventory model under function principle, Information Science, 95(1996)(1-2), 71-79.

[2] S.H. Chen and C.H. Hsieh, Graded mean integration representations of generalized fuzzy number, Journal of Chinese Fuzzy Systems, 5(1999), 1-7.

[3] G. Hadley and T. M. Whitin, Analysis of Inventory Systems, Prentice-Hall, Englewood clipps, NJ, 1963.

[4] F. Harris, Operations and Cost, AW Shaw Co. Chicago, 1915.

[5] R. Jain, Decision making in the presence of fuzzy variables, IIIE Transactions on systems:Man and Cybernetics, 17( 1976), 698-703.

[6] J. Kacpryzk and P. Staniewski, Long-term inventory policy-making through fuzzy-decision making models, Fuzzy Sets and Systems, 8(1982), 117-132.

[7] Muhammad Al-Salamah, Economic production quantity in an imperfect manufacturing process with synchronous and asynchronous flexible rework rates, Operations $R e$ search Perspectives, 6(2019)1000103.

[8] K. S. Park, Fuzzy set theoretical interpretation of economic order quantity, IEEE Trans. Systems Man. Cybernet SMC, 17(1987), 1082-1084.
[9] W. Ritha and K. Kalaiarasi, Optimization of fuzzy integrated Vendor-Buyer inventory models, Annala of Fuzzy Mathematics and Informatics, 2(2)(2011), 239-257.

[10] W. Ritha and K. Kalaiarasi, Optimization of fuzzy integrated two stage Vendor-Buyer inventory system, International of Mathematical Sciences and Applications, 1(2)(2011), 1-12.

[11] W. Ritha and K. Kalaiarasi, Optimization of a multiple vendor single Buyer integrated inventory model with a variable number of vendors, International Journal of Mathematical Sciences and Engineering Applications, 5(V)(2011), 173-188.

[12] G. Urgeletti Tinarelli, Inventory control models and problems, European Journal of Operational Research, 14(1983), 1-12.

[13] R. Wilson, A scientific routine for stock control, Harvard Business Review, 13(1934), 116-128.

[14] L. A. Zadeh, Fuzzy sets, Information Control, 8(1965), 338-353.

[15] L. A. Zadeh and R. E. Bellman, Decision making in a fuzzy environment, Management Science, 17(1970), 140164.

[16] H. J. Zimmerman, Using fuzzy sets in operational research, European Journal of Operational Research, 13(1983), 201-206.

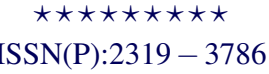

Malaya Journal of Matematik $\operatorname{ISSN}(\mathrm{O}): 2321-5666$ 